\title{
LOCAL CONVEXITY OF METRIC BALLS
}

\author{
PARISA HARIRI, RIKU KLÉN, AND MATTI VUORINEN
}

\begin{abstract}
We study local convexity properties of the triangular ratio metric balls in proper subdomains of the real coordinate space. We also study inclusion properties of the visual angle metric balls and related hyperbolic type metric balls in the complement of the origin and the upper half space.
\end{abstract}

\section{INTRODUCTION}

The hyperbolic metric has become an important tool in geometric function theory. It works well in simply connected subdomains of the complex plane, because we can use the Riemann mapping theorem to map such domains onto the unit disk, where explicit formulas are known [B1, [KL]. In higher dimensions $(n \geq 3)$ no counterpart exists and thus there is need for other methods. One approach is to generalize the hyperbolic metric for higher dimensions in such a manner that the generalized metric is comparable with the hyperbolic metric when the domain is the upper half plane or the unit ball

$$
\mathbb{H}^{n}=\left\{\left(x_{1}, \ldots, x_{n}\right) \in \mathbb{R}^{n}: x_{n}>0\right\}, \quad \mathbb{B}^{n}=\left\{z \in \mathbb{R}^{n}:|z|<1\right\} .
$$

We call these generalizations hyperbolic type metrics. One of the first hyperbolic type metrics, the quasihyperbolic metric, was introduced by Gehring and Palka in the 1970's GP. Soon the quasihyperbolic metric found numerous applications and nowadays it is a standard tool in geometric function theory, see e.g. $[\mathrm{GH}]$. During the past two decades many authors have introduced various other hyperbolic type metrics, [HIMPS], [KLVW], [B2], [S], HMM.

However, it is not clear which one of these hyperbolic type metrics is preferable for a specific application. The natural line of research in this situation is to compare the geometries defined by two hyperbolic type metrics to each other. In this paper we study so called triangular ratio metric or $s$-metric, which is defined as follows for a domain $G \subset \mathbb{R}^{n}$ and $x, y \in G$ :

$$
s_{G}(x, y)=\sup _{z \in \partial G} \frac{|x-y|}{|x-z|+|z-y|} \in[0,1] .
$$

This metric has been studied in [CHKV, HKLV]. For a metric space $(G, m)$ we define the metric ball for $x \in G$ and $r>0$ by $B_{m}(x, r)=\{y \in G$ : $m(x, y)<r\}$. We study the metric balls defined by the triangular ratio metric. The behaviour of a metric can be studied in many different ways.

File: hkv20171108.tex, printed: 2021-8-2, 6.08

2010 Mathematics Subject Classification. 51M10(52A20).

Key words and phrases. triangular ratio metric ball, visual angle metric, local convexity. 
Our goal is to examine the geometric properties of the metric space $\left(G, s_{G}\right)$ by discussing local convexity properties of the metric balls $B_{s_{G}}(x, r)$. We prove that $B_{s_{G}}(x, r), r \in(0,1]$, is always starlike with respect to $x$ and find the best constant $r_{0}$ such that $B_{s_{G}}(x, r), r \in\left(0, r_{0}\right)$, is convex. Similar local convexity results for other hyperbolic type metrics can be found in [HKLV, K1, K2, K3, KRT, HPS]. We also compare different hyperbolic type metrics by considering the inclusion of metric balls. For some other hyperbolic type metrics similar research is carried out in [KV1, KV2].

We next formulate our main results.

Theorem 1.2. Let $G \subsetneq \mathbb{R}^{n}$ be a domain. Then for all $z \in G$ and all $r \in(0,1]$, the $s$-ball $B_{s}(z, r)$ is Euclidean starlike with respect to $z$.

In addition to the $s$-metric, we also study other metrics defined on subdomains of $\mathbb{R}^{n}$, for example the quasihyperbolic metric $k_{G}$, the distance ratio metric $j_{G}$, the visual angle metric $v_{G}$, and the point pair function $p_{G}$ which are defined in Section 4. For these metrics we summarize our results in the following theorems.

Theorem 1.3. Let $x \in \mathbb{R}^{n} \backslash\{0\}, r \in\left(0, r_{m}\right]$, and $m \in\{j, k,|\cdot|, p, q, s\}$. Then we can find the best possible radius $t=t(r)$ such that $B_{m}(x, t) \subset B_{v}(x, r)$.

Theorem 1.4. Let $x \in \mathbb{H}^{n}$ and $r \in(0, \pi / 2]$. Then

$$
\begin{aligned}
B^{n}\left(x+\left(\sec ^{2} r-1\right) x_{n} e_{n},(\tan r) x_{n}\right) & \subset B_{v}(x, r) \\
& \subset B^{n}\left(x+\left(2 x_{n} \tan ^{2} r\right) e_{n}, 2 x_{n} \frac{\tan r}{\cos r}\right),
\end{aligned}
$$

and the Euclidean balls are the best possible. Moreover

$$
B^{n}\left(x, x_{n} \sin r\right) \subset B_{v}(x, r) \subset B^{n}\left(x, 2 x_{n}\left(\frac{\tan r}{\cos r}+\tan ^{2} r\right)\right) .
$$

This paper may be considered to be a continuation of the earlier studies [HKLV, K1, K2, K3, KMS. Our main results and their proofs suggest that similar results might be valid for other metrics as well and this offers ideas for further studies of the same topic, for instance for the Apollonian or the Seittenranta metrics [B2, S].

\section{Starlikeness AND CONVEXity of triangular RATIO METRIC BALLS}

In this section we consider local convexity properties of $s$-metric balls. We start with $\mathbb{R}^{n} \backslash\{0\}$ and generalize the results to proper subdomains of $\mathbb{R}^{n}$. Before studying local convexity properties we introduce preliminary results.

Given two points $x$ and $y$ in $\mathbb{R}^{n}$, the line segment between them is denoted by

$$
[x, y]=\{(1-t) x+t y: 0 \leq t \leq 1\},
$$

and $\measuredangle(x, z, y)$ stands for the angle in the range $[0, \pi]$ between the line segments $[x, z]$ and $[y, z]$.

Definition 2.1. Let $G \subsetneq \mathbb{R}^{n}$ be a domain and $x \in G$. We say that $G$ is starlike with respect to $x$ if for every $y \in G,[x, y] \subset G$. The domain $G$ is strictly starlike with respect to $x$ if $G$ is bounded and each ray from $x$ meets $\partial G$ at exactly one point. 
Proof of Theorem 1.2. Without loss of generality we may assume $z=0$. Fix $0<r \leq 1$. Let $y \in B_{s}(0, r)$. Since $s(y, 0)<1,[0, y] \subset G$. Let $x \in[0, y]$ and $z \in \partial G$. Since

$$
|x| \leq|z|+|x-z|,|x|+|x-y|=|x| \text {, and }|y-z| \leq|y-x|+|x-z|,
$$

we have

$$
\begin{aligned}
\frac{|x|}{|z|+|x-z|} & \leq \frac{|x|+|x-y|}{|z|+|x-z|+|x-y|}=\frac{|y|}{|z|+|x-z|+|x-y|} \\
& \leq \frac{|y|}{|z|+|y-z|} \leq s(y, 0) .
\end{aligned}
$$

Taking a supremum over all $z \in \partial G$ we thus obtain

$$
s(x, 0) \leq s(y, 0)<r,
$$

since $x$ is an arbitrary point in $[0, y] \subset B_{s}(0, r)$.

Next we continue the study of [HKLV] by considering the convexity of triangular ratio metric balls in a general subdomain of $\mathbb{R}^{n}$.

Lemma 2.2. [HKLV, 3.6, 3.8] Let $x \in G=\mathbb{R}^{n} \backslash\{0\}$ and $r \in(0,1)$. Then $B_{s}(x, r)$ is (strictly) convex if and only if $r \leq 1 / 2(r<1 / 2)$.

Theorem 2.3. Let $G \subsetneq \mathbb{R}^{n}$ be a domain, $x \in G$ and $r \in(0,1)$. Then $B_{s}(x, r)$ is convex if $r \leq 1 / 2$.

Proof. By [HKLV], (2.2)] the ball $B_{s}(x, r)$ is the intersection of balls $B_{s_{z}}(x, r)$, where $s_{z}$ is the triangular ratio metric in $\mathbb{R}^{n} \backslash\{z\}, z \in \partial G$, and by Lemma 2.2 each of these balls $B_{s_{z}}(x, r)$ is convex. The assertion follows as intersection of convex domains is a convex domain.
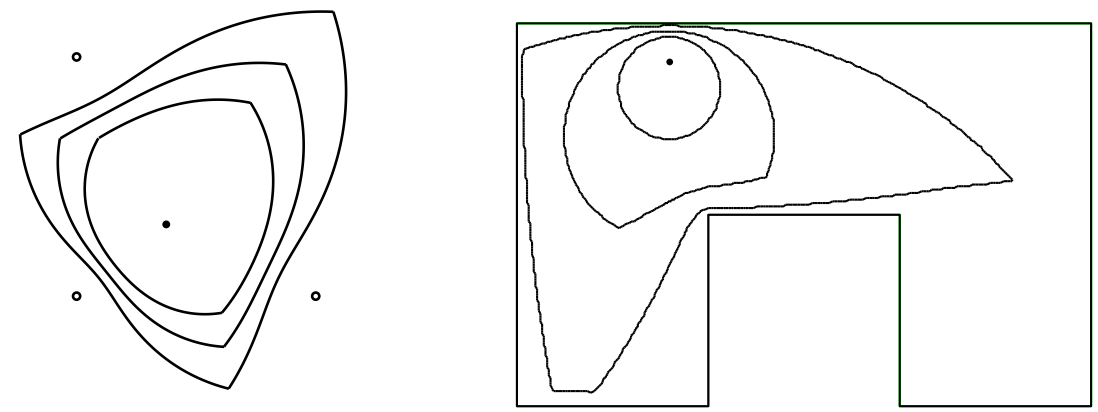

FiguRE 1. Left: The $s$-metric disks $s\left(0.75 e_{1}+0.6 e_{2}, r\right)$ in $\mathbb{R}^{2} \backslash\left\{0,2 e_{1}, 2 e_{2}\right\}$ with $r=0.4, r=0.5, r=0.6$. Right: $s$ metric disks in a polygonal domain.

Finally, we make the following conjecture for the balls of the point pair function, which is defined below in 4.4 .

Conjecture 2.4. Let $x \in \mathbb{R}^{n} \backslash\{0\}$ and $r \in(0,1)$. Then $B_{p}(x, r)$ is (strictly) convex if and only if $r \leq \sqrt{2}-1$. 


\section{Formula For Visual ANGLe Metric IN $\mathbb{H}^{n}$}

For a domain $G \subsetneq \mathbb{R}^{n}, n \geq 2$, and $x, y \in G$ let

$$
v_{G}(x, y)=\sup \{\measuredangle(x, z, y): z \in \partial G\} .
$$

If $\partial G$ is not a proper subset of a line, then $v_{G}$ defines a metric on $G$, as shown in [KLVW, Lemma 2.8].

The supremum in (3.1) can be found by a geometric construction if $G=$ $\mathbb{B}^{2}$. Indeed by [KLVW] Theorem 1.2], by considering the two points $z_{1}$ and $z_{2}$ of intersection of the ellipses with foci at $0, x$ and $0, y$, respectively, both with focal sum equal to 1 , the formula for $v_{\mathbb{B}^{2}}(x, y)$ is just

$$
v_{\mathbb{B}^{2}}(x, y)=\max \left\{\measuredangle\left(x, z_{1} /\left|z_{1}\right|, y\right), \measuredangle\left(x, z_{2} /\left|z_{2}\right|, y\right)\right\} .
$$

Here we find an analogue of this formula for $\mathbb{H}^{2}$ by finding the points of intersection of two parabolas with foci at $x$ and $y$, respectively, and both with the real axis $\partial \mathbb{H}^{2}$ as the directrix, see Figure 2 .

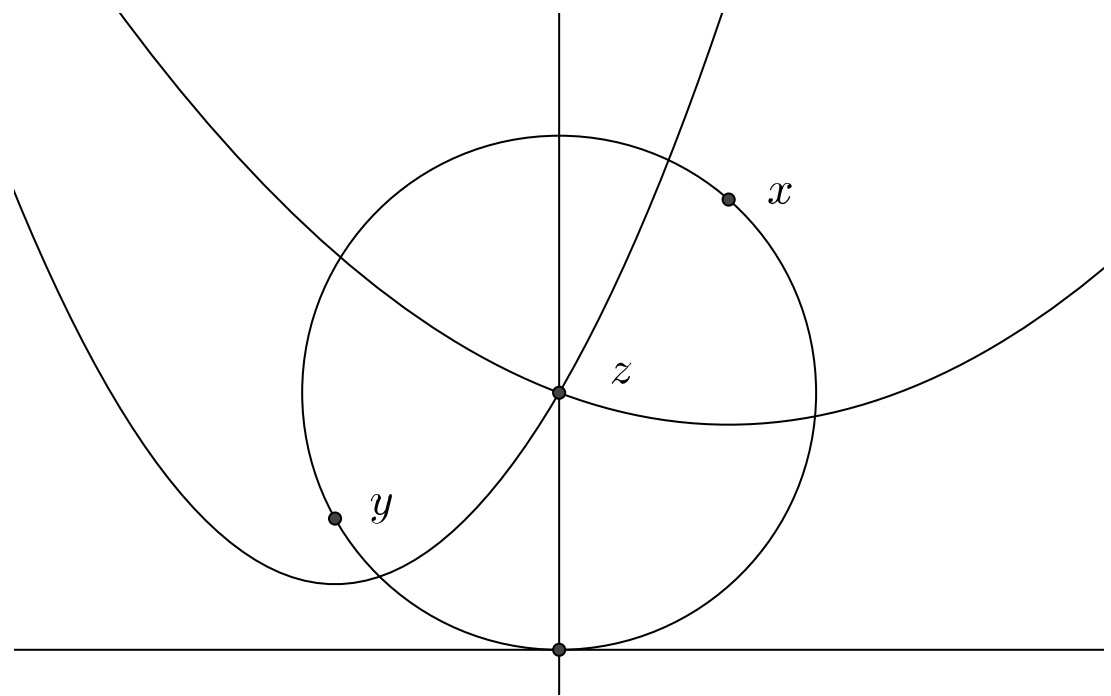

FigURE 2. The point $z=\left(z_{1}, z_{2}\right)$ is the intersection of the parabola with focus $x$ and directrix $\partial \mathbb{H}^{2}$ and the parabola with focus $y$ and directrix $\partial \mathbb{H}^{2}$. The extremal point $\left(z_{1}, 0\right)$ in the definition of $v_{\mathbb{H}^{2}}(x, y)$ can be found as the projection of $z$ to $\partial \mathbb{H}^{2}$.

For this purpose it is convenient to use horocycles. For two distinct points $x, y \in G$ where $G=\mathbb{B}^{2}$ or $G=\mathbb{H}^{2}$, a horocycle through $x, y$ is a Euclidean circle or line through $x$ and $y$ tangent to $\partial G$.

We consider the problem of finding the center points of two horocycles. These centers are the points of intersection of the parabolas with foci at $x$ 
and $y$ and directrix $\partial \mathbb{H}^{2}$. Therefore the formula for centers $z=\left(z_{1}, z_{2}\right)$ of these horocycles are given by

$$
|x-z|=z_{2}=|y-z|,
$$

we have

$$
\left\{\begin{array}{l}
\left(x_{1}-z_{1}\right)^{2}+\left(x_{2}-z_{2}\right)^{2}=z_{2}{ }^{2}, \\
\left(y_{1}-z_{1}\right)^{2}+\left(y_{2}-z_{2}\right)^{2}=z_{2}{ }^{2} .
\end{array}\right.
$$

By solving this system of quadratic equations we get

$$
z_{1}=\frac{x_{2} y_{1}-x_{1} y_{2} \pm \sqrt{x_{2} y_{2}}|x-y|}{x_{2}-y_{2}}, \quad x_{2} \neq y_{2} .
$$

If $x_{2}=y_{2}$, then $z_{1}=\left(x_{1}+y_{1}\right) / 2$. In terms of this solution in the case $x_{2} \neq y_{2}$, the possible extremal points for the visual angle metric are the two possible points of the form $\left(z_{1}, 0\right) \in \partial \mathbb{H}^{2}$, and here we choose either + or whichever corresponds to the smaller imaginary part.

Now by Figure 3

$$
\begin{gathered}
v_{\mathbb{H}^{2}}(x, y)=\pi-\alpha-\beta, \\
\alpha=\arctan \left(\frac{x_{2}}{z_{1}-x_{1}}\right) \text { and } \beta=\arctan \left(\frac{y_{2}}{y_{1}-z_{1}}\right) .
\end{gathered}
$$

Therefore

$$
v_{\mathbb{H}^{2}}(x, y) \equiv \pi-\arctan \left(\frac{2 \sqrt{x_{2} y_{2}}|x-y| \pm\left(x_{2}+y_{2}\right)\left(x_{1}-y_{1}\right)}{\left(x_{1}-y_{1}\right)^{2}-4 x_{2} y_{2}}\right) \quad(\bmod \pi) .
$$

Another formula for $v_{\mathbb{H}^{2}}(x, y)$ can be derived from

$$
z_{2}=\frac{|x-y|}{2\left(x_{2}-y_{2}\right)^{2}}\left(|x-y|\left(x_{2}+y_{2}\right) \mp 2\left(x_{1}-y_{1}\right) \sqrt{x_{2} y_{2}}\right)
$$

and the law of cosines together with the inscribed angle theorem.

\section{INCLUSION PROPERTIES OF METRIC BALlS IN $\mathbb{R}^{n} \backslash\{0\}$ AND $\mathbb{H}^{n}$}

In this section we study inclusions of the visual angle metric balls and other metric balls. We begin by defining the metrics which we use.

4.1. Quasihyperbolic metric. Let $G$ be a proper subdomain of $\mathbb{R}^{n}$. For all $x, y \in G$, the quasihyperbolic metric $k_{G}$ is defined as

$$
k_{G}(x, y)=\inf _{\gamma} \int_{\gamma} \frac{1}{d(z, \partial G)}|d z|,
$$

where the infimum is taken over all rectifiable $\operatorname{arcs} \gamma$ joining $x$ to $y$ in $G$ GP]. If we assume $x, y \in G=\mathbb{R}^{n} \backslash\{0\}$ and the angle $\varphi$ between the line segments $[0, x]$ and $[0, y]$ satisfies $0<\varphi<\pi$ then by [Vu2, 3.11]

$$
k_{G}(x, y)=\sqrt{\varphi^{2}+\log ^{2} \frac{|x|}{|y|}}, \quad G=\mathbb{R}^{n} \backslash\{0\} .
$$

4.3. Distance ratio metric. For a proper open subset $G \subset \mathbb{R}^{n}$ and for all $x, y \in G$, the distance ratio metric $j_{G}$ is defined as

$$
j_{G}(x, y)=\log \left(1+\frac{|x-y|}{\min \{d(x, \partial G), d(y, \partial G)\}}\right) .
$$




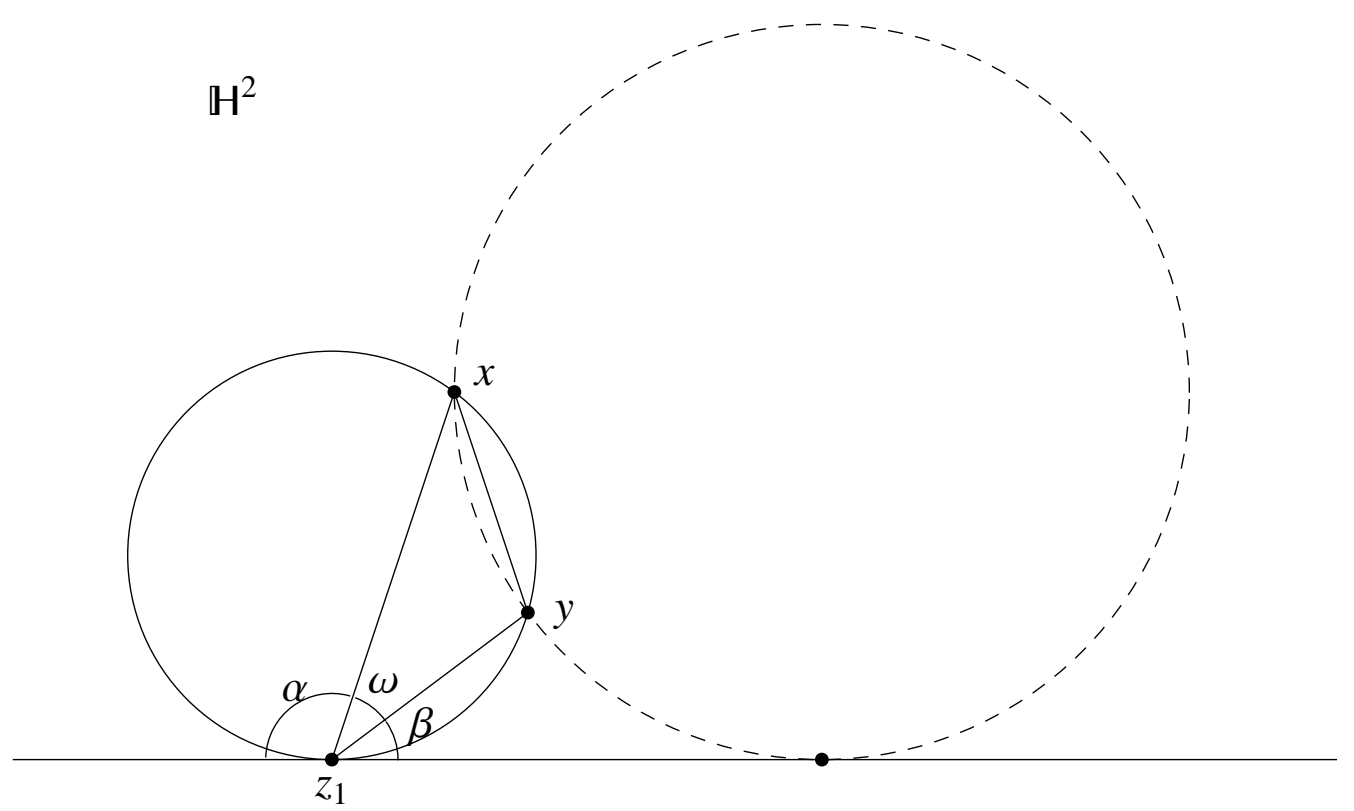

Figure 3. Two horocycles through $x$ and $y$ and the extremal point $\left(z_{1}, 0\right)$ with $v_{\mathbb{H}^{2}}(x, y)=\measuredangle\left(x, z_{1}, y\right)$.

This metric was introduced by Gehring and Palka [GP] in a slightly different form and in the above form in [Vu1]. If confusion seems unlikely, then we also write $d(x)=d(x, \partial G)$.

4.4. Point pair function. We define for $x, y \in G \subsetneq \mathbb{R}^{n}$ the point pair function

$$
p_{G}(x, y)=\frac{|x-y|}{\sqrt{|x-y|^{2}+4 d(x) d(y)}} .
$$

This point pair function was introduced in [CHKV] where it turned out to be a very useful function in the study of the triangular ratio metric. However, there are domains $G$ such that $p_{G}$ is not a metric: for instance this is the case if $G=\mathbb{B}^{2}$, CHKV, Remark 3.1].

4.5. Chordal metric. The chordal metric is defined by

$$
\left\{\begin{array}{l}
q(x, y)=\frac{|x-y|}{\sqrt{1+|x|^{2}} \sqrt{1+|y|^{2}}} \quad ; x, y \in \mathbb{R}^{n}, \\
q(x, \infty)=\frac{1}{\sqrt{1+|x|^{2}}} .
\end{array}\right.
$$

Proposition 4.6. If $G=\mathbb{R}^{n} \backslash\{0\}$, then the $v$-balls $B_{v}(x, r), x \in G, r \in$ $(0, \pi)$, are angular domains with vertex at 0 .

Lemma 4.7. For all $x \in \mathbb{R}^{n} \backslash\{0\}, r \in(0, \pi]$

$$
B_{s}\left(x, \sin \frac{r}{2}\right) \subset B_{v}(x, r),
$$

and the radius $\sin \frac{r}{2}$ is best possible. 
Proof. By symmetry we may assume that $n=2$ and $x=e_{1}$. Let $y=$ $t e^{i r}, t>0$. Now

$$
s_{G}(x, y)=\frac{|x-y|}{|x|+|y|}=\frac{\sqrt{1+t^{2}-2 t \cos r}}{1+t}=: f(t) .
$$

By Proposition 4.6, we want to minimize $f(t)$. Now

$$
f^{\prime}(t)=\frac{(t-1)(1+\cos r)}{(1+t)^{2} \sqrt{1+t^{2}-2 t \cos r}},
$$

and $f^{\prime}(t)=0$ if and only if $t=1$, so $|y|=|x|$ and $s_{G}(x, y)=\frac{|x-y|}{2|x|}=\sin \frac{r}{2}$. The sharpness follows from this argument.

Lemma 4.8. For all $x \in \mathbb{R}^{n} \backslash\{0\}$ and $r \in(0, \pi]$

$$
B_{j}\left(x, \log \left(1+2 \sin \frac{r}{2}\right)\right) \subset B_{v}(x, r),
$$

and the radius $\log \left(1+2 \sin \frac{r}{2}\right)$ is best possible.

Proof. In the same way as in the proof of Lemma 4.7, we may assume again that $n=2, x=e_{1}$ and $y=t e^{i r}, t>0$. Now

$$
j_{G}(x, y)=\log \left(1+\frac{|x-y|}{\min \{|x|,|y|\}}\right)=\log \left(1+\frac{\sqrt{1+t^{2}-2 t \cos r}}{\min \{1, t\}}\right) .
$$

Define

$$
f(t)= \begin{cases}\log \left(1+\frac{\sqrt{1+t^{2}-2 t \cos r}}{t}\right) & \text { for } t \leq 1, \\ \log \left(1+\sqrt{1+t^{2}-2 t \cos r}\right) & \text { for } t>1 .\end{cases}
$$

Computation yields

$$
f^{\prime}(t)= \begin{cases}\frac{t \cos r-1}{t\left(1-2 t \cos r+t\left(t+\sqrt{1+t^{2}-2 t \cos r}\right)\right.} & \text { for } t \leq 1, \\ \frac{t-\cos r}{1+t^{2}-2 t \cos r+\sqrt{1+t^{2}-2 t \cos r}} & \text { for } t>1 .\end{cases}
$$

By Proposition 4.6, the extremal case takes place when $t=1$, and hence $|y|=|x|$ and $j_{G}(x, y)=\log \left(1+\frac{|x-y|}{|x|}\right)=\log \left(1+2 \sin \frac{r}{2}\right)$. Therefore $R=$ $\log \left(1+2 \sin \frac{r}{2}\right)$ and the proof is complete. The sharpness follows from this proof.

Lemma 4.9. For all $x \in \mathbb{R}^{n} \backslash\{0\}$ and $r \in(0, \pi]$

$$
B_{k}(x, r) \subset B_{v}(x, r),
$$

and the radius $r$ is best possible.

Proof. We assume by symmetry that $n=2, x=t e_{1}$ and $y=e_{1}, t>0$. Now

$$
k_{G}(x, y)=\sqrt{r^{2}+\log ^{2} \frac{|x|}{|y|}}=\sqrt{r^{2}+\log ^{2} t}=: f(t),
$$


and

$$
f^{\prime}(t)=\frac{\log t}{t \sqrt{r^{2}+\log ^{2} t}} .
$$

By Proposition 4.6, the extremal case happens when $t=1$, so $|y|=|x|$ and $k_{G}(x, y)=r$ and the proof is complete. The sharpness follows from the proof.

Lemma 4.10. For all $x \in \mathbb{R}^{n} \backslash\{0\}$ and $r \in(0, \pi]$, we have

$$
\mathbb{B}^{n}(x, R) \subset B_{v}(x, r), \quad R= \begin{cases}|x| \sin r & \text { for } r \in(0, \pi / 2], \\ |x| & \text { for } r \in(\pi / 2, \pi],\end{cases}
$$

and the radius $R$ is best possible.

Proof. Fix $x \in \mathbb{R}^{n} \backslash\{0\}$, and $y \in B_{v}(x, r)$. We consider two cases. If $r \in(\pi / 2, \pi]$ then by Proposition $4.6,|x-y| \leq|x|$. If $r \in(0, \pi / 2]$ then by the law of sines and Proposition 4.6 $|x-y| \leq|x| \sin r$. The sharpness follows from the proof.

Lemma 4.11. For all $x \in \mathbb{R}^{n} \backslash\{0\}$ and $r \in(0, \pi]$, we have

$$
B_{q}(x, R) \subset B_{v}(x, r), \quad R=\min \left\{\frac{2|x| \sin r / 2}{1+|x|^{2}}, \frac{|x|}{\sqrt{1+|x|^{2}}}\right\},
$$

and the radius $R$ is best possible.

Proof. Fix $x \in \mathbb{R}^{n} \backslash\{0\}$, and $y \in B_{v}(x, r)$. We consider two cases. If $r \in(\pi / 2, \pi]$ then

$$
q(x, y) \leq q(x, 0)=\frac{|x|}{\sqrt{1+|x|^{2}}} .
$$

If $r \in(0, \pi / 2]$ we may assume that $x=e_{1}$, and let $y=t e^{i r}, t>0$. Then by the law of sines

$$
q(x, y)=\frac{|x-y|}{\sqrt{1+|x|^{2}} \sqrt{1+|y|^{2}}}=\frac{\sqrt{1+t^{2}-2 t \cos r}}{\sqrt{2} \sqrt{1+t^{2}}}=: f(t),
$$

and

$$
f^{\prime}(t)=\frac{\left(t^{2}-1\right) \cos r}{\sqrt{2}\left(1+t^{2}\right)^{3 / 2} \sqrt{1+t^{2}-2 t \cos r}} .
$$

The extremal case takes place when $t=1$, therefore $|x|=|y|$, and

$$
q(x, y)=\frac{|x-y|}{1+|x|^{2}}=\frac{2|x| \sin (r / 2)}{1+|x|^{2}} .
$$

The proof is complete because by Proposition 4.6, $B_{v}(x, y)$ is an angular domain. The sharpness follows from the proof.

Lemma 4.12. For all $x \in \mathbb{R}^{n} \backslash\{0\}$ and $r \in(0, \pi]$, we have

$$
B_{p}(x, R) \subset B_{v}(x, r), \quad R=\frac{\sin (r / 2)}{\sqrt{\sin ^{2}(r / 2)+1}},
$$

and the radius $R$ is best possible. 
Proof. By symmetry we may assume that $n=2$ and $x=e_{1}$. Let $y=$ $t e^{i r}, t>0$. By the definition

$$
p_{G}(x, y)=\frac{|x-y|}{\sqrt{|x-y|^{2}+4|x||y|}}=\sqrt{\frac{1+t^{2}-2 t \cos r}{1+t^{2}-2 t \cos r+4 t}}=: f(t) .
$$

By Proposition 4.6, we want to minimize $f(t)$. Now

$$
f^{\prime}(t)=\frac{2\left(t^{2}-1\right)}{\sqrt{1+t^{2}-2 t \cos r}(1+t(4+t)-2 t \cos r)^{3 / 2}},
$$

and $f^{\prime}(t)=0$ if and only if $t=1$, so $|y|=|x|$ and

$$
p_{G}(x, y)=\frac{|x-y|}{\sqrt{|x-y|^{2}+4|x|^{2}}}=\frac{\sin (r / 2)}{\sqrt{\sin ^{2}(r / 2)+1}} .
$$

The sharpness follows from the proof.

Proof of Theorem 1.3. The proof follows from Lemmas $4.7-4.12$.

Theorem 4.13. For all $x \in \mathbb{H}^{n}$ and $r \in(0, \pi / 2)$, we have

$$
\begin{aligned}
B_{v}(x, r) & \subset B^{n}\left(x+\left(2 T^{2}\right) x_{n} e_{n}, 2 T\left(\sqrt{T^{2}+1}\right) x_{n}\right) \\
& \subset B^{n}\left(x,\left(2 T \sqrt{T^{2}+1}+2 T^{2}\right) x_{n}\right),
\end{aligned}
$$

where $T=|\tan (\pi-r)|$. Moreover, the smaller Euclidean ball is the smallest possible containing $B_{v}(x, r)$.

Note that 4.14 is equivalent to

$$
\begin{aligned}
B_{v}(x, r) & \subset B^{n}\left(x+\left(2 x_{n} \tan ^{2} r\right) e_{n}, 2 x_{n} \frac{\tan r}{\cos r}\right) \\
& \subset B^{n}\left(x, 2 x_{n}\left(\frac{\tan r}{\cos r}+\tan ^{2} r\right)\right) .
\end{aligned}
$$

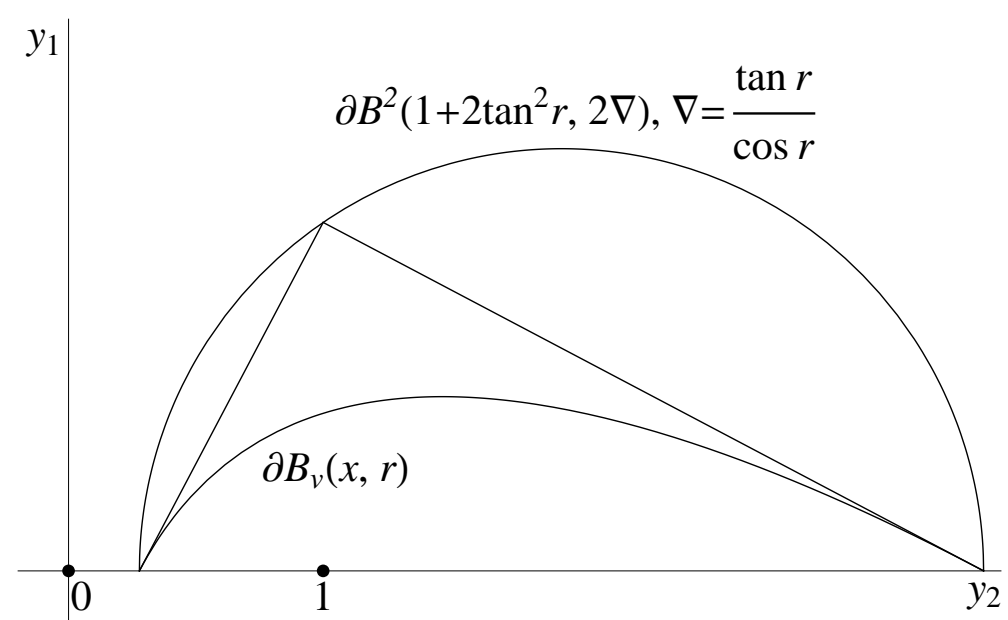

Figure 4. Proof of Theorem 4.13 
Proof. It suffices to consider the case $n=2$. For the first inclusion, let us fix $x=i$. We claim that

$$
B_{v}(i, r) \subset B^{2}\left(\left(1+2 \tan ^{2} r\right) i, 2 \frac{\tan r}{\cos r}\right) .
$$

By $(3.2)$, we have

$$
v_{\mathbb{H}^{2}}(x, y) \equiv \pi-\arctan \left(\frac{2 \sqrt{y_{2}} \cdot \sqrt{y_{1}^{2}+\left(y_{2}-1\right)^{2}} \mp y_{1}\left(1+y_{2}\right)}{y_{1}^{2}-4 y_{2}}\right) \quad(\bmod \pi) .
$$

Writing $v_{\mathbb{H}^{2}}(i, y)=r$, we conclude that

$$
y_{1}=\left\{\begin{array}{l}
\cot r\left(1+y_{2}-2 \sqrt{y_{2}} \sec r\right)=: f_{1}\left(y_{2}, r\right), \\
\cot r\left(-1-y_{2}+2 \sqrt{y_{2}} \sec r\right)=: f_{2}\left(y_{2}, r\right) .
\end{array}\right.
$$

This gives the equation of $\partial B_{v}(i, r)$, for $y_{2} \in\left[b_{1}, b_{2}\right]$.

Letting $f_{1}\left(y_{2}, r\right)=f_{2}\left(y_{2}, r\right)=0$, we see that

$$
y_{2}=\left\{\begin{array}{l}
2(1-\sin r) \sec ^{2} r-1=: b_{1}, \\
2(1+\sin r) \sec ^{2} r-1=: b_{2} .
\end{array}\right.
$$

Our next goal is to find the equation of $\partial B^{2}\left(\left(1+2 \tan ^{2} r\right) i, 2 \frac{\tan r}{\cos r}\right)$. Taking $\left|y-\left(1+2 \tan ^{2} r\right)\right|=2 \frac{\tan r}{\cos r}$, gives

$$
y_{1}=\left\{\begin{array}{l}
-\sqrt{4 y_{2} \sec ^{2} r-\left(1+y_{2}\right)^{2}}=: g_{1}\left(y_{2}, r\right), \\
\sqrt{4 y_{2} \sec ^{2} r-\left(1+y_{2}\right)^{2}}=: g_{2}\left(y_{2}, r\right) .
\end{array}\right.
$$

By symmetry, it is sufficient to show that $g_{2}\left(y_{2}, r\right) \geq f_{2}\left(y_{2}, r\right)$. In order to prove this inequality, it is convenient to estimate the circular $\operatorname{arc} g_{2}\left(y_{2}, r\right)$, by a triangle with vertices $b_{1}, b_{2}$ and $\left(1, g_{2}(1, r)\right)$. We can do this estimation because

$$
\frac{\partial^{2}}{\partial y_{2}{ }^{2}}\left(g_{2}\left(y_{2}, r\right)\right)=\frac{-4 \sec ^{2} r \tan ^{2} r}{\left(4 y_{2} \sec ^{2} r-\left(1+y_{2}\right)^{2}\right)^{3 / 2}} \leq 0 .
$$

Denote the above mentioned triangle by $T\left(y_{2}, r\right)$

$$
T\left(y_{2}, r\right)= \begin{cases}y_{1}=\frac{g_{2}(1, r)}{1-b_{1}}\left(y_{2}-b_{1}\right)=: l_{1}\left(y_{2}, r\right), & b_{1} \leq y_{2}<1 \\ y_{1}=\frac{g_{2}(1, r)}{1-b_{2}}\left(y_{2}-b_{2}\right)=: l_{2}\left(y_{2}, r\right), & 1 \leq y_{2} \leq b_{2}\end{cases}
$$

We only need to show that $h\left(y_{2}, r\right)=T\left(y_{2}, r\right)-f_{2}\left(y_{2}, r\right) \geq 0$.

If $b_{1} \leq y_{2}<1$, then

$$
h\left(y_{2}, r\right)=\frac{1}{\cos r}\left(y_{2}-1+\left(y_{2}+1\right) \sin r\right)-\cot r\left(2 \sqrt{y_{2}} \sec r-1-y_{2}\right) .
$$

But

$$
\frac{\partial^{2}\left(h\left(y_{2}, r\right)\right)}{\partial y_{2}^{2}}=\frac{\csc r}{2 y_{2}^{3 / 2}}>0, \text { for } 0 \leq r \leq \pi / 2 .
$$

Hence $h^{\prime}\left(y_{2}, r\right)$ is an increasing function of $y_{2}$. Moreover we claim that indeed

$$
h^{\prime}\left(b_{1}, r\right)=\cot r+\sec r+\tan r-\frac{\csc r}{\sqrt{-1+2 /(1+\sin r)}}=0 .
$$


To see this, it is sufficient to make the following observation

$$
\sqrt{\frac{1-\sin r}{1+\sin r}}=\frac{\csc r}{\cot r+\sec r+\tan r} .
$$

Now it is easy to check that for $0 \leq r \leq \pi / 2$, both sides are equivalent to $\csc \left(\frac{\pi}{4}+\frac{r}{2}\right) \sin \left(\frac{\pi}{4}-\frac{r}{2}\right)$. Thus $h^{\prime}\left(y_{2}, r\right) \geq 0$.

Similarly we can show that

$$
h\left(b_{1}, r\right)=2 \sec r\left(\csc r-1-\cot r \sqrt{\frac{1-\sin r}{1+\sin r}}\right)=0 .
$$

To see this, it suffices to show that

$$
\frac{1-\sin r}{\cos r}=\sqrt{\frac{1-\sin r}{1+\sin r}} .
$$

It follows easily that for $0 \leq r \leq \pi / 2$, both sides are equivalent to

$$
\csc \left(\frac{\pi}{4}+\frac{r}{2}\right) \sin \left(\frac{\pi}{4}-\frac{r}{2}\right) \text {. }
$$

Hence $h\left(y_{2}, r\right) \geq 0$. In the same manner for $1 \leq y_{2} \leq b_{2}$,

$$
h\left(y_{2}, r\right)=\left(1+y_{2}+\csc r-y_{2} \csc r\right) \tan r-\cot r\left(-1-y_{2}+2 \sec r \sqrt{y_{2}}\right) \text {. }
$$

But

$$
\frac{\partial^{2}\left(h\left(y_{2}, r\right)\right)}{\partial y_{2}^{2}}=\frac{\csc r}{2 y_{2}^{3 / 2}}>0, \text { for } 0 \leq r \leq \pi / 2
$$

and

$$
h^{\prime}\left(b_{2}, r\right)=\tan r+\cot r-\sec r-\frac{\csc r}{\sqrt{2 \sec ^{2} r+2 \tan r \sec r-1}}=0 .
$$

To see this, it is enough to show that

$$
\sqrt{2 \sec ^{2} r+2 \tan r \sec r-1}=\frac{\csc r}{\tan r+\cot r-\sec r},
$$

and it is easy to check that for $0 \leq r \leq \pi / 2$, both sides are equivalent to $\csc \left(\frac{\pi}{4}-\frac{r}{2}\right) \sin \left(\frac{\pi}{4}+\frac{r}{2}\right)$. Therefore $h^{\prime}\left(y_{2}, r\right) \geq 0$. We next show that

$$
h\left(b_{2}, r\right)=2 \sec r(\csc r-\cot r \sqrt{2 \sec r(\tan r+\sec r)-1}+1)=0 .
$$

To see this we need to show that

$$
\sqrt{2 \sec r(\tan r+\sec r)-1}=(1+\csc r) \tan r .
$$

In the same manner as in the previous part, we can show that for $0 \leq r \leq$ $\pi / 2$, both sides are equivalent to $\csc \left(\frac{\pi}{4}-\frac{r}{2}\right) \sin \left(\frac{\pi}{4}+\frac{r}{2}\right)$. Hence $h\left(y_{2}, r\right) \geq$ 0 .

An easy computation shows that $g_{2}\left(b_{1}, r\right)=g_{2}\left(b_{2}, r\right)=0$. Hence the Euclidean ball $B^{2}\left(\left(1+2 \tan ^{2} r\right) i, 2 \frac{\tan r}{\cos r}\right)$ is the smallest possible ball containing $B_{v}(x, r)$, and this completes the proof for the first inclusion.

For the second inclusion, let

$$
y \in \partial B^{2}\left(x+\left(2 x_{2} \tan ^{2} r\right) e_{2}, 2 \frac{\tan r}{\cos r} x_{2}\right) .
$$


It follows that

$$
\left|y-x_{1} e_{1}-\left(1+2 \tan ^{2} r\right) x_{2} e_{2}\right| \leq 2 \frac{\tan r}{\cos r} x_{2} .
$$

Therefore

$$
\begin{aligned}
|y-x| & \leq\left|y-x_{1} e_{1}-\left(1+2 \tan ^{2} r\right) x_{2} e_{2}\right|+\left|-x+x_{1} e_{1}+\left(1+2 \tan ^{2} r\right) x_{2} e_{2}\right| \\
& \leq 2 x_{2}\left(\frac{\tan r}{\cos r}+\tan ^{2} r\right),
\end{aligned}
$$

and $y \in \partial B^{2}\left(x, 2 x_{2}\left(\frac{\tan r}{\cos r}+\tan ^{2} r\right)\right)$.

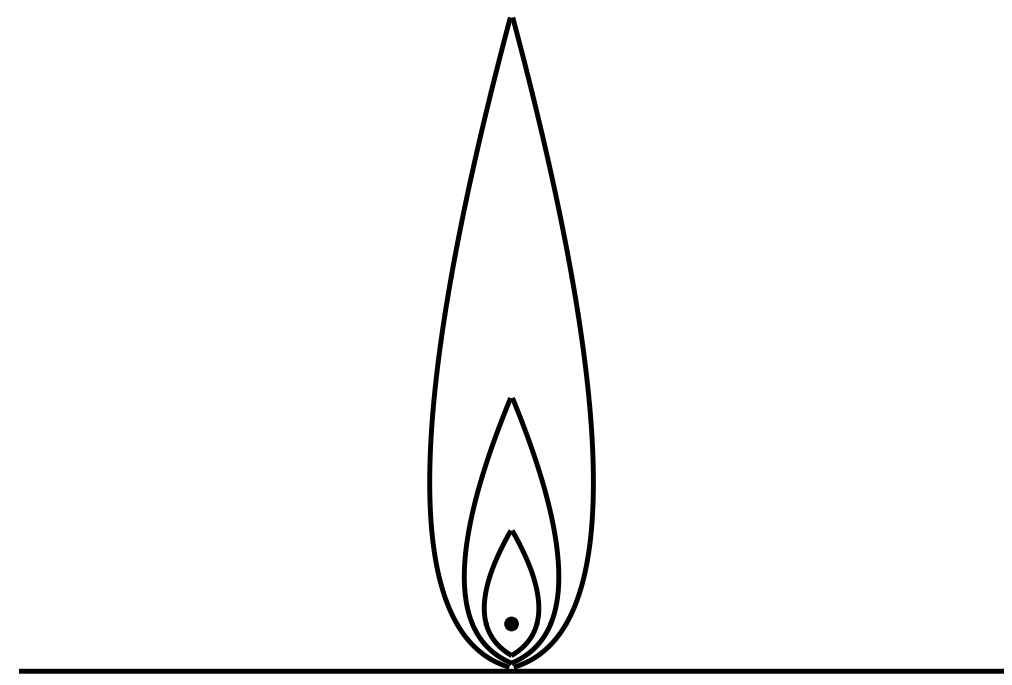

FiguRE 5. $\partial B_{v}(i, r)$, in the upper half space, for $r=\pi / 6, \pi / 4, \pi / 3$.

Remark 4.18. By the proof of Theorem 4.13 we observe that $\partial B_{v}(x, r)$ is not smooth for $0 \leq r \leq \pi$. By 4.15),

$$
f_{2}^{\prime}\left(b_{1}^{+}, r\right)=\cot r\left(\frac{\sec r}{\sqrt{2 \sec ^{2} r-2 \tan r \sec r-1}}-1\right),
$$

and

$$
f_{1}^{\prime}\left(b_{1}^{+}, r\right)=-\cot r\left(\frac{\sec r}{\sqrt{2 \sec ^{2} r-2 \tan r \sec r-1}}-1\right) .
$$

Hence we see that at the point $y_{2}=b_{1}$, the derivative does not exist and $\partial B_{v}(x, r)$ is not smooth.

Remark 4.19. The proof of Theorem 4.13 gives more, namely, the lines $l_{1}\left(y_{2}, r\right)$ and $l_{2}\left(y_{2}, r\right)$ are tangent to the $\partial B_{v}(x, r)$. To see this we first compute the slope of the tangent lines to $B_{v}(x, r)$ at the points $b_{1}, b_{2}$. We have by Remark 4.18

$$
f_{2}^{\prime}\left(b_{1}^{+}, r\right)=\cot r\left(\frac{\sec r}{\sqrt{2 \sec ^{2} r-2 \tan r \sec r-1}}-1\right) .
$$


Next, by 4.17), the slope of the line $l_{1}$ is

$$
m_{1}=\frac{\tan r}{-\sec ^{2} r+\tan r \sec r+1} .
$$

We claim that $m_{1}=f_{2}^{\prime}\left(b_{1}^{+}, r\right)$. To see this it is enough to show that

$$
\frac{\tan ^{2} r+1-\sec ^{2} r+\sec r \tan r}{\sec r}=\frac{-\sec ^{2} r+\tan r \sec r+1}{\sqrt{2 \sec ^{2} r-2 \tan r \sec r-1}} .
$$

It is easy to check that for $0 \leq r \leq \pi / 2$, both sides are equivalent to

$$
\cos \left(\frac{r}{2}\right) \csc \left(\frac{\pi}{4}-\frac{r}{2}\right) \csc \left(\frac{\pi}{4}+\frac{r}{2}\right) \sin \left(\frac{r}{2}\right) .
$$

Similarly, substituting $b_{2}$ from (4.16), gives

$$
f_{2}^{\prime}\left(b_{2}^{+}, r\right)=\cot r\left(\frac{\sec r}{\sqrt{2 \sec ^{2} r+2 \tan r \sec r-1}}-1\right) .
$$

Next, by (4.17), the slope of line $l_{2}$ is

$$
m_{2}=-\frac{\tan r}{\sec ^{2} r+\tan r \sec r-1} .
$$

We claim that $m_{2}=f_{2}^{\prime}\left(b_{2}^{+}, r\right)$. To see this, it is enough to show that

$$
\frac{-\tan ^{2} r-1+\sec ^{2} r+\sec r \tan r}{\sec r}=\frac{\sec ^{2} r+\tan r \sec r-1}{\sqrt{2 \sec ^{2} r+2 \tan r \sec r-1}} .
$$

It is easy to check that for $0 \leq r \leq \pi / 2$, both sides are equivalent to

$$
\cos \left(\frac{r}{2}\right) \csc \left(\frac{\pi}{4}-\frac{r}{2}\right) \csc \left(\frac{\pi}{4}+\frac{r}{2}\right) \sin \left(\frac{r}{2}\right) .
$$

Theorem 4.20. For all $x \in \mathbb{H}^{n}$ and $r \in(0, \pi / 2)$, we have

$$
B^{n}\left(x+\left(\sec ^{2} r-1\right) x_{n} e_{n},(\tan r) x_{n}\right) \subset B_{v}(x, r) .
$$

Proof. By symmetry, it suffices to consider the case $n=2$.

Let us fix $x=i$. We claim that

$$
B^{2}\left(i \sec ^{2} r, \tan r\right) \subset B_{v}(x, r) .
$$

By 4.15), the equation of $\partial B_{v}(i, r)$ is as follows:

$$
y_{1}=\left\{\begin{array}{l}
\cot r\left(1+y_{2}-2 \sqrt{y_{2}} \sec r\right)=: f_{1}\left(y_{2}, r\right), \\
\cot r\left(-1-y_{2}+2 \sqrt{y_{2}} \sec r\right)=: f_{2}\left(y_{2}, r\right) .
\end{array}\right.
$$

Next we find the equation of $\partial B^{2}\left(i \sec ^{2} r, \tan r\right)$. Taking $\left|y-\sec ^{2} r\right|=\tan r$ gives

$$
y_{1}=\left\{\begin{array}{l}
-\sqrt{-\left(y_{2}-\sec ^{2} r\right)^{2}+\tan ^{2} r}=: g_{1}\left(y_{2}, r\right), \\
\sqrt{-\left(y_{2}-\sec ^{2} r\right)^{2}+\tan ^{2} r}=: g_{2}\left(y_{2}, r\right) .
\end{array}\right.
$$

By symmetry, it is sufficient to show that $f_{2}\left(y_{2}, r\right) \geq g_{2}\left(y_{2}, r\right)$. To see this we only need to show that $h\left(y_{2}, r\right)=: f_{2}\left(y_{2}, r\right)^{2}-g_{2}\left(y_{2}, r\right)^{2} \geq 0$. But

$$
\frac{\partial^{2} h\left(y_{2}, r\right)}{\partial y_{2}^{2}}=\frac{1}{y_{2}^{3 / 2}}\left(2 y_{2}^{3 / 2}+\cos r-3 y_{2} \cos r\right) \csc ^{2} r \text {. }
$$

Denote by

$$
h_{2}\left(y_{2}, r\right)=2 y_{2}^{3 / 2}+\cos r-3 y_{2} \cos r .
$$


But

$$
\frac{\partial^{2} h_{2}\left(y_{2}, r\right)}{\partial y_{2}^{2}}=\frac{3}{2 \sqrt{y_{2}}}>0, \text { and } \quad \frac{\partial h_{2}\left(\cos ^{2} r, r\right)}{\partial y_{2}}=0 .
$$

Moreover $h_{2}\left(\cos ^{2} r, r\right)>0$ for $r \in(0, \pi / 2)$. Therefore $\frac{\partial^{2} h\left(y_{2}, r\right)}{\partial y_{2}^{2}} \geq 0$. It is easy to check that $\frac{\partial h\left(\sec ^{2} r, r\right)}{\partial y_{2}}=0$, and hence $h\left(y_{2}, r\right) \geq 0$.

Theorem 4.21. For all $x \in \mathbb{H}^{n}$ and $r \in(0, \pi / 2)$, we have

$$
B^{n}\left(x, x_{n} \sin r\right) \subset B_{v}(x, r) .
$$

Proof. Let $\lambda=\sin r$ and $y \in B^{n}\left(x, \lambda x_{n}\right)$. By domain monotonicity of the $v$-metric, we have for $B_{x}=B^{n}\left(x, x_{n}\right)$ the inequalities

$$
v_{\mathbb{H}^{n}}(x, y) \leq v_{B_{x}}(x, y) \leq \arcsin \lambda,
$$

where the last inequality follows by [KLVW, 3.3].

Proof of Theorem 1.4. The proof follows from Theorems $4.13,4.20$ and 4.21 .

Acknowledgements. The research of the first author was supported by UTUGS, The Graduate School of the University of Turku.

\section{REFERENCES}

[AVV] G. D. Anderson, M. K. Vamanamurthy, and M. Vuorinen, Conformal invariants, inequalities and quasiconformal maps. J. Wiley, 1997.

[B1] A. F. BeARdon, The geometry of discrete groups. Graduate texts in math., Vol. 91, Springer-Verlag, New York, 1983.

[B2] A. F. BeArdon, The Apollonian metric of a domain in $\mathbb{R}^{n}$. Quasiconformal mappings and analysis (Ann Arbor, MI, 1995), 91-108, Springer, New York, 1998.

[CHKV] J. Chen, P. Hariri, R. Klén, and M. Vuorinen, Lipschitz conditions, triangular ratio metric, and quasiconformal maps. Ann. Acad. Sci. Fenn. 40 (2015), 683-709.

[GH] F. W. Gehring And K. HaG, The Ubiquitous Quasidisk, Mathematical Surveys and Monographs 184, Amer. Math. Soc., Providence, RI, 2012.

[GP] F. W. Gehring and B. P. PAlKa, Quasiconformally homogeneous domains. J. Analyse Math. 30 (1976), 172-199.

[HIMPS] P. Hästö, Z. Ibragimov, D. Minda, S. Ponnusamy and S. K. Sahoo, Isometries of some hyperbolic-type path metrics, and the hyperbolic medial axis, In the tradition of Ahlfors-Bers, IV, Contemporary Math. 432 (2007), 63-74.

[HMM] D. A. Herron, W. Ma, And D. Minda, Möbius invariant metrics bilipschitz equivalent to the hyperbolic metric. Conform. Geom. Dyn. 12 (2008), 67-96.

[HKLV] S. Hokuni, R. KlÉn, Y. Li, And M. Vuorinen, Balls in the triangular ratio metric. Proceedings of an international conference, Complex Analysis and Dynamical Systems VI,Contemp. Math. Volume 667, 2016, 105-123.

[HPS] P. Hästö, S. Ponnusamy, and S. K. Sahoo, Inequalities and geometry of the Apollonian and related metrics. Rev. Roumaine Math. Pures Appl. 51 (2006), 433-452.

[KL] L. KeEn And N. LAKIC, Hyperbolic geometry from a local viewpoint. London Mathematical Society Student Texts, 68. Cambridge University Press, Cambridge, 2007. $\mathrm{x}+271 \mathrm{pp}$.

[K1] R. KLÉN, Local convexity properties of quasihyperbolic balls in punctured space. J. Math. Anal. Appl. 342 (2008), 192-201.

[K2] R. KLÉn, Close-to-convexity of quasihyperbolic and j-metric balls. Ann. Acad. Sci. Fenn. Math. 35 (2010), 493-501. 
[K3] R. KLÉN, Local convexity properties of balls in Apollonian and Seittenranta's metrics. Conform. Geom. Dyn. 17 (2013), 133-144.

[KLVW] R. Klén, H. Lindén, M. Vuorinen, and G. WAng, The visual angle metric and Möbius transformations. Comput. Methods Funct. Theory 14 (2014), 577608.

[KMS] R. Klén, M. R. Mohapatra, and S. K. Sahoo, Geometric properties of the Cassinian metric. Math. Nachr. 290 (2017), 1531-1543.

[KRT] R. Klén, A. Rasila, and J. TAlponen, Quasihyperbolic geometry in Euclidean and Banach spaces. J. Anal. 18 (2010), 261-278.

[KV1] R. KLÉN AND M. VuORINEn, Inclusion relations of hyperbolic type metric balls. Publ. Math. Debrecen 81 (2012), 289-311.

[KV2] R. KLÉN AND M. VUORINEn, Inclusion relations of hyperbolic type metric balls II. Publ. Math. Debrecen 83 (2013), 21-42.

[S] P. Seittenranta, Möbius-invariant metrics. Math. Proc. Cambridge Philos. Soc. 125 (1999), 511-533.

[Vu1] M. Vuorinen, Conformal invariants and quasiregular mappings. J. Anal. Math. 45 (1985), 69-115.

[Vu2] M. VuORINEn, Conformal geometry and quasiregular mappings. Lecture notes in math. 1319, Springer-Verlag, Berlin, 1988.

[Vu3] M. Vuorinen, Geometry of Metrics. Proc. ICM2010 Satellite Conf. International Workshop on Harmonic and Quasiconformal Mappings (HMQ2010), eds. D. Minda, S. Ponnusamy, N. Shanmugalingam, J. Analysis 18 (2010), 399-424, ISSN 0971-3611. http://arxiv.org/abs/1101.4293.

Department of Mathematics and Statistics, University of Turku, Turku, FinLAND

E-mail address: parisa.hariri@utu.fi

Department of Mathematics and Statistics, University of Turku, Turku, FINLAND

E-mail address: riku.klen@utu.fi

Department of Mathematics and Statistics, University of Turku, Turku, FINLAND

E-mail address: vuorinen@utu.fi 